\title{
Spiders' mechanical lifelines provide a key for the study of trust in the quality of materials
}

\begin{abstract}
Shigeyoshi Osaki
It is very important for living creatures to have trust in most of the environments encountered in daily life. However, there have been no reports evaluating the degree of trust in such environments, including the quality of materials. The mechanical properties of a spider dragline may be considered an appropriate object for studying trust in the quality of materials such as fibers, textiles and plastics, because a spider must trust its life to the dragline when falling. In this study, the stress-strain curves of a dragline, and the effects of ultraviolet rays on its mechanical properties, were found to present an origin for evaluating the degree of trust on the quality of materials. Hypothetically, spiders may have trust in only the linear region of the stress-strain curve, where there is no possibility of the dragline breaking; they may have no confidence in the nonlinear region where there is the risk of breakage. Thus, the degree of trust could be estimated by establishing whether there is a linear relationship between stimulus and response and by determining the slope of the straight line. The concept of trust developed here is applicable to specifications for various industrial materials and also to social phenomena.
\end{abstract}

Polymer Journal (2011) 43, 194-199; doi:10.1038/pj.2010.122; published online 8 December 2010

Keywords: industrial materials; mechanical property; spider silk; stimulus response; trust

\section{INTRODUCTION}

It is impossible for living organisms to function without some degree of trust in their environment, where dangers are frequently encountered. Problems related to safety and trust have recently attracted considerable attention in the complicated society of high technology. Trust has been studied not only as the quality of industrial materials but also as economic and psychological issues in social sciences. ${ }^{1-5}$ To deal with trust quantitatively is very difficult because the subtleties of trust come from the fact that trust is a mental state that cannot be measured directly. To my knowledge, there have been no reports on methods for evaluating trust based on natural sciences. It is of tremendous importance to understand the real nature of trust scientifically from the viewpoint of effectively avoiding dangers and making a living with safety. However, it has been very difficult to understand the real nature of trust scientifically because no appropriate methods have been found for evaluating trust numerically. Here, the author focuses on a spider's mechanical lifeline, which its total weight is perfectly applied to. Spiders necessarily use their draglines as their mechanical lifeline controlling life and death when jumping to capture insect prey on their orb webs or falling to escape a predator. He paid attention to the mechanical properties of spider silks under different environmental conditions because the mechanical functions of not only lifeline but also silks constituting orb webs of diurnal spiders for capturing insect prey outdoors could have been markedly affected by exposure to ultraviolet (UV) irradiation. The spider places its complete trust in the lifeline under such an environmental condition. However, it is doubtful for spiders to perfectly trust in all functions of their silks under sunlight. Thus, it is very important to study which part of the lifeline the spider trusts under their environmental condition, such as at night and in the daytime, from the viewpoint of understanding an origin of trust.

Much research has focused on the physicochemical and aging properties of spider draglines, ${ }^{6-11}$ including studies on mechanical properties such as the mechanical breaking stress (BS) and the elastic modulus. ${ }^{12-18}$ However, most researchers only focused on such studies based on natural science. In my previous work, I put forth a concept of maximal safety from a mechanical lifeline of spiders. ${ }^{19}$ It was found that the elastic limit strength (ELS) of a dragline was approximately twice that of the mature spider's weight, ${ }^{19-21}$ so that the dragline could be safely used as a mechanical lifeline.

As the ELS of draglines is related to safety, it may also be connected with trust. Although it is of great interest to study how living organisms trust their environment, there have been no reports to date proposing methods for evaluating such trust. In a previous paper, ${ }^{22}$ it was found that spiders usually use only part of the limiting mechanical characteristics. This author focuses on the mechanical properties of a spider dragline, which he considers to be an appropriate object for studying the concept of trust, under environments at night and in the daytime.

In the present study, the aim of the author was to probe the nature of trust and then provide a clue for dealing with trust in the quality of industrial materials quantitatively. Here, a method has been developed 
for evaluating the degree of trust from a mechanical perspective, and is applied to specifications for mechanical properties of various industrial materials, and also to social phenomena.

\section{MATERIALS AND METHODS}

Spiders

The spiders used here were mainly female Nephila clavata. Female Leucauge blanda and Argiope bruennichii spiders were also used. These spiders were mature and diurnal and were active in the daytime outdoors.

\section{Sampling}

Orb webs are mechanically supported by a main frame of radial threads, which are similar to the draglines that function as 'mechanical lifelines' for falling spiders by supporting their bodyweight. ${ }^{19,20}$ Although radial threads should be used for studying the effects of UV rays on orb webs, it is not appropriate to use radial threads cut directly from built orb webs as samples because of the mechanical hysteresis associated with cutting and building. Thus, it is appropriate to estimate the mechanical strength of the orb web by using draglines instead of radial threads, as both types of threads are secreted from the same ampullate gland. ${ }^{9}$

Live spiders were used in the experiments just after being captured from orb webs. We obtained long draglines from a spider by sticking draglines to both sides of a plastic frame $\sim 50 \mathrm{~cm}$ long covered with adhesive when falling from a wooden bar under laboratory conditions. No extra stress above the elastic limit stress (ES) was applied to avoid mechanical hysteresis, ${ }^{20-22}$ and the draglines were cut into eight specimens and stuck to both sides of a paper frame covered with adhesive for mechanical measurements. One of the eight specimens was assigned for scanning electron microscope observation and the other specimens were used for UV irradiation during fixed periods. We repeated the sampling more than five times using live spiders.

In a previous paper, ${ }^{21}$ the ratio of spider's weight to the cross-section of draglines was determined to be almost constant. We judge the homogeneity of draglines from the values of the ratio of the spider's weight to the cross-section.

We ascertained whether the samples were true draglines consisting of double filaments using a scanning electron microscope (JEOL, Tokyo, Japan), ${ }^{17,20}$ before the stress-strain curves were measured. The cross-sectional area of draglines was determined using a scanning electron microscope. The draglines consisted almost entirely of protein, with an amino acid composition mainly comprising glycine, alanine and glutamic acid residues. ${ }^{23}$

\section{Mechanical measurements, UV irradiation}

The stress-strain curves for dragline samples $4 \mathrm{~cm}$ in effective length, which were ascertained to be true draglines, were measured using a modified TENSILON UTM-IIIL (A \& D Ltd., Tokyo, Japan) with a stretching velocity of $3.3 \times 10^{-4} \mathrm{~m} \mathrm{~s}^{-1}, 11,17,20$ which is relatively low compared with the actual velocity of secretion. The mechanical measurements of draglines taken immediately after irradiation of UV rays were taken five times, using samples prepared from a dragline of the same spider. Here, ELS is determined from ES multiplied by the cross-sectional area of the dragline. ES is defined as the stress at the elastic limit point, where the stress-strain behavior changes from linear to nonlinear. The mechanical breaking strength is determined from the BS, which is defined as the stress at the breaking point of the dragline multiplied by the cross-section of the dragline. These data obtained from five samples were averaged and the s.d. was determined.

UV rays reaching the ground from the sun are mainly UV-A rays (320$400 \mathrm{~nm}$ ) as very small intensity of UV-B rays $(290-320 \mathrm{~nm})$ and no UV-C rays $(270-290 \mathrm{~nm})$ reach the earth's surface. UV rays with wavelengths longer than $270 \mathrm{~nm}$ and an intensity distribution similar to UV-A and UV-B rays were created by a $660 \mathrm{~W}$ xenon-arc lamp (Suntester XF-180, Shimadzu, Japan) with a special filter, thereby yielding an intensity distribution similar to sunlight. ${ }^{11}$ An air fan was installed in the UV irradiation source to avoid heating of samples. In the UV-A* irradiation instrument, samples were set $25 \mathrm{~cm}$ from the xenon-arc lamp. UV-A* and UV-B*, which were irradiated to samples, were prepared by excluding wavelengths shorter than $320 \mathrm{~nm}$ and $290 \mathrm{~nm}$, respectively, from the xenon-arc lamp using special filters. UV-B* contains UV-B and UV-A rays, whereas UV-A* contains only UV-A rays. The intensity of the
$\mathrm{UV}^{-A^{*}}$ rays was similar to that of natural UV-A rays, whereas the intensity of the UV- $\mathrm{B}^{\star}$ rays, which increase by breaking the ozone layer, was approximately double that of natural UV-B rays.

\section{RESULTS}

Trust in the mechanical properties of the lifeline

It is of great interest to study the mechanical behaviors of draglines for understanding which part of the draglines spiders trust in. Stressstrain curves were measured for eight specimens prepared from a dragline sample secreted from a female $N$. clavata (Japanese golden web spider) spider weighing $540 \mathrm{mg}$ at the same time. The strain was found to increase linearly with an increase in stress between the origin and the elastic limit point, but nonlinearly between the elastic limit point and the breaking point. All the specimens showed almost identical behavior in the linear region, where the stress-strain relationship was linear and reversible. Figure 1 shows stress-strain curves for three different specimens prepared from the dragline. The straight line indicates that strain can be accurately estimated for a given stress, following Hooke's law, and the slope of the straight line is related to the elastic modulus reflecting rigidity in a very small strain. Generally, the length of the linear region and its slope depend on the mechanical functions of the spider's dragline, which is affected by the spider's growth. For example, the length of the linear region reflects that of the elastic region, whereas its slope reflects the degree of rigidity. Anyway, spiders could hang by their draglines in safety in the linear region. As the stress-strain curve in the linear region shows a reversible behavior, there is no possibility of breaking, and spiders can precisely estimate the mechanical functions of draglines in the same way as the weighing machine with normal spring, which follows Hooke's law. However, the specimens showed marked fluctuations in mechanical behaviors in the nonlinear region (see Figure 1) where the stress-strain relationship was irreversible. Most of the protein molecules constituting draglines are mainly amorphous, whereas only a part of the protein molecules forms $\beta$-configuration (a pleated sheet form with intramolecular bonds). ${ }^{24-26}$ Furthermore, the entanglements among protein molecules exist in the amorphous state as the molecular chains are very long. The entanglements may be helpful for exhibiting the elasticity of draglines when applied stress is not more than the ES. However, stress above the ES causes loosening in the entanglements of protein molecules, which cannot be reconstructed in the amorphous

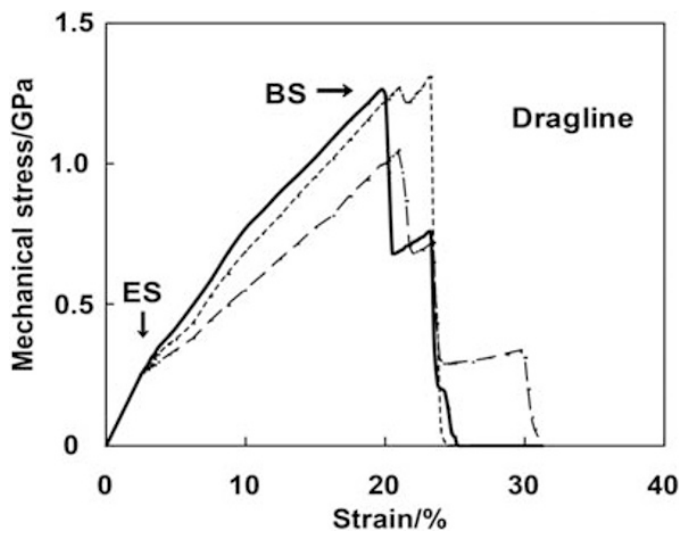

Figure 1 Stress-strain curves for three different specimens of draglines secreted from a Nephila clavata spider weighing $540 \mathrm{mg}$. The stress-strain behavior changes from linear to nonlinear at the elastic limit point. Elastic limit stress (ES) and breaking stress (BS) are defined, respectively, as the stress at the elastic limit point at which the stress-strain behavior changes from linear to nonlinear, and as the breaking stress at the breaking point. 


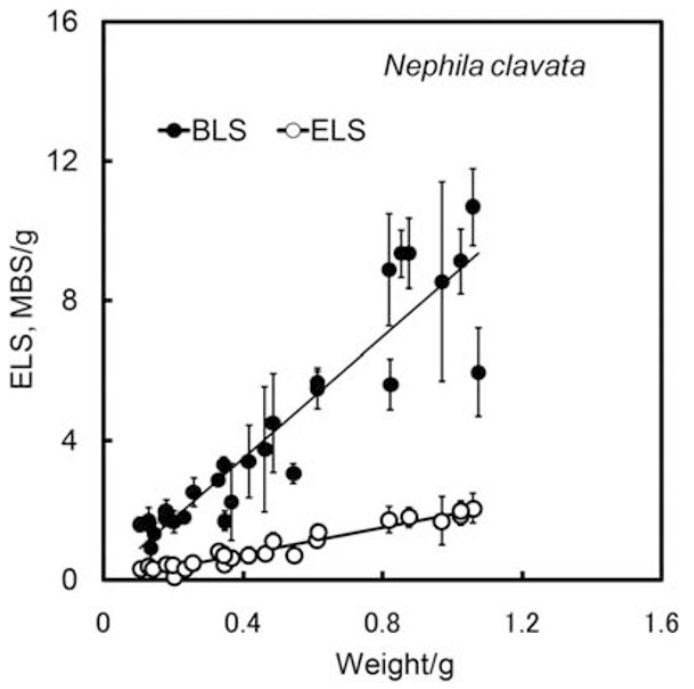

Figure 2 The elastic limit strength (ELS) and the mechanical breaking strength (MBS) are plotted against different weights of spiders. The ELS and the MBS are defined, respectively, as the strength at the elastic limit point at which the force-elongation behavior of draglines of Nephila clavata spiders changes from linear to nonlinear and as the strength at the breaking point. A variation in ELS and MBS is shown as s.d.

state, even after the stress has been removed from the dragline. This irreversibility may be responsible for the nonlinear behavior observed above the ES.

Generally, it is very dangerous for spiders to act freely without exact recognition of the actual limit of their mechanical functions. ${ }^{22}$ To compare the maximum limits in linear and nonlinear regions of mechanical functions of draglines, here, the mechanical breaking strength and ELS were plotted against the spider's weight in Figure 2. Variation in mechanical breaking strength was found to be very large for draglines of spiders with different weights, whereas the variation in ELS was extremely small (see Figure 2). Therefore, it was very difficult to accurately estimate stress-strain behavior in the nonlinear region, or the limit point at which the draglines would break. More than two BS peaks were observed in each stress-strain curve, as shown in Figure 1, which correspond to the double filaments constituting the dragline ${ }^{18-20}$ and also to fibrillation of filaments. Spiders could not trust the nonlinear region, as they could not estimate exactly when and where the dragline would break. In contrast, the ELS increases linearly with the spider's weight and it follows a law that the ELS is twice the spider's weight. ${ }^{19,20}$ Thus, spiders could recognize the limit of the linear region and could then have complete confidence in the linear region where the draglines were mechanically safe.

Therefore, the degree of trust could be evaluated by checking whether the relationship of stress-strain showed a linear or nonlinear behavior, and by determining the slope of the straight line. As the slope is directly related to the elastic modulus, the larger slope mechanically presents a higher degree of trust for spiders. On the other hand, the length of the linear region reflects the length of the period that spiders can mechanically trust the dragline for. These may be a hereditary result of the spider's long evolutionary history.

\section{Effects of UV irradiation on Lifeline}

Draglines from diurnal $N$. clavata spiders are irradiated by UV rays from the sun in the daytime. Therefore, it is of great importance to study the mechanical behaviors of draglines for understanding how a
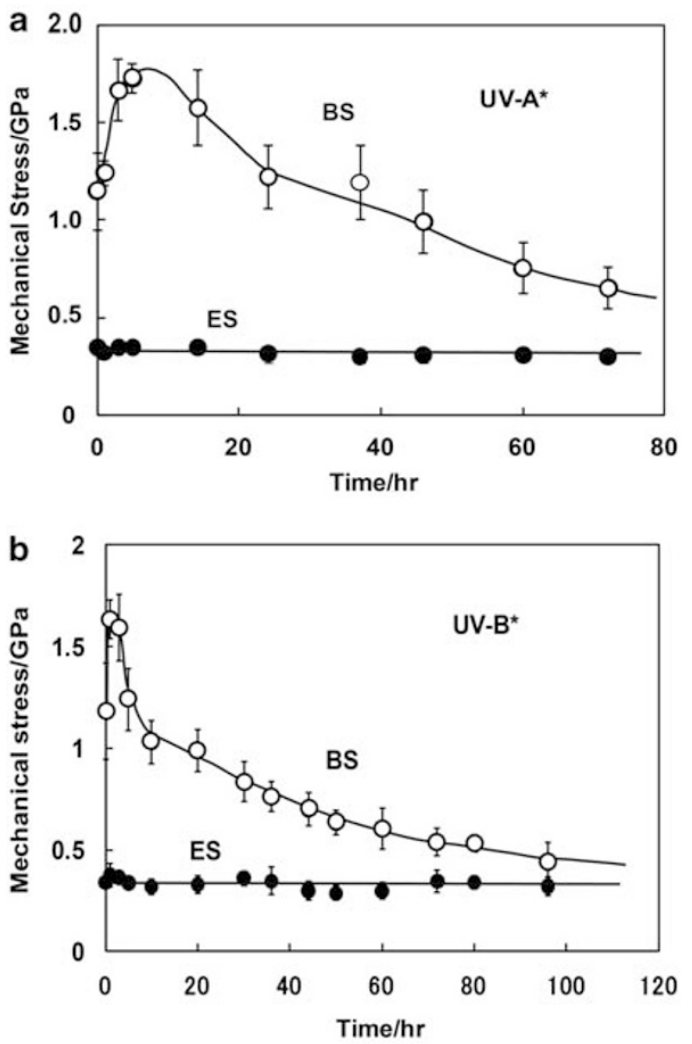

Figure 3 (a) Time dependence of ultraviolet UV-A* irradiation on the breaking stress (BS) and elastic limit stress (ES) of draglines (mean value \pm s.e.) secreted from a female Nephila clavata spider weighing $540 \mathrm{mg}$. Closed circles represent ES, and open circles represent BS. The UV-A* rays were prepared by excluding wavelengths shorter than $320 \mathrm{~nm}$ from a xenon-arc lamp. (b) Time dependence of UV-B* irradiation on the BS and ES of draglines (mean value \pm s.e.) secreted from a Nephila clavata spider weighing $540 \mathrm{mg}$. Closed circles represent ES, and open circles represent $\mathrm{BS}$. The UV-B* rays were prepared by excluding wavelengths shorter than $290 \mathrm{~nm}$ from a xenon-arc lamp.

spider trusts her dragline as a lifeline under the strict condition of UV irradiation. The effects of UV rays on the mechanical properties of the dragline were investigated, to further explore the notion of trust. Figures $3 \mathrm{a}$ and $\mathrm{b}$ show the time-dependent effect of UV irradiation on the BS and ES of draglines secreted from a female $N$. clavata spider weighing $540 \mathrm{mg}$. The $\mathrm{UV}^{-} \mathrm{A}^{*}$ and $\mathrm{UV}^{*} \mathrm{~B}^{\star}$ rays used here were prepared by excluding wavelengths shorter than 320 and $290 \mathrm{~nm}$, respectively, from a xenon-arc lamp. The BS increased rapidly after commencing UV-A* or UV-B* irradiation, peaked fairly quickly, then decreased gradually as it approached an asymptote. The asymptotic value was lower at the starting point and was apt to approach the value of ES with time. BS approached the value of ES more rapidly under $\mathrm{UV}^{-} \mathrm{B}^{*}$ irradiation than under $\mathrm{UV}-\mathrm{A}^{*}$ irradiation, as the UV-B ${ }^{*}$ rays were more destructive to silk proteins compared with UV- $A^{\star}$ rays (see Figure 2 of ref. 11). The s.e. in the data of ES after UV-B ${ }^{\star}$ irradiation is a little larger than that after $U V-A^{*}$ irradiation, even though the s.e. in the data of ES is much smaller than that in BS. Thus, the BS was strongly affected by UV-A ${ }^{*}$ or UV-B ${ }^{\star}$ irradiation, ${ }^{11}$ and prolonged exposure increased the possibility of breaking.

As the intensity of UV irradiation from sunlight is dependent on weather, spiders could not predict the useful value of the mechanical function of draglines and also when and where the draglines would break. 


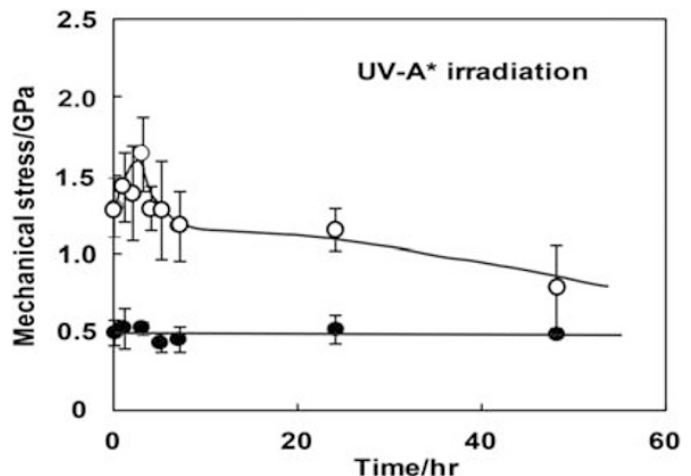

Figure 4 Time dependence of ultraviolet UV-A* irradiation on the breaking stress (BS) and elastic limit stress (ES) of draglines (mean value \pm s.e.) secreted from a female Leucauge blanda spider weighing $71 \mathrm{mg}$. Closed circles represent ES, and open circles represent BS. UV-A* rays were prepared by excluding wavelengths shorter than $320 \mathrm{~nm}$ from a xenon-arc lamp.

$\mathrm{UV}^{-} \mathrm{A}^{*}$ irradiation also produced a peak in normalized BS for a female $L$. blanda weighing $70 \mathrm{mg}, 27$ then decreased gradually and tended to approach the value of ES, whereas ES was almost constant even after UV-A* irradiation, as shown in Figure 4. The time dependence of UV-A* irradiation on BS also produced a small peak, but the ES was almost constant for the period investigated, although the results of UV-A* irradiation to A. bruennichii spiders are not shown here. Such peaks in the BS shown in Figures 3a, b and 4 may be mainly a consequence of two factors: an increase in molecular weight ${ }^{28-30}$ due to crosslinking of protein molecules, ${ }^{11}$ and a decrease in molecular weight due to decomposition. ${ }^{31}$ Although the effects of generating crosslinks are significant for a period after the commencement of UV irradiation, the effects of chemical decomposition become dominant over time. ${ }^{29}$ The peaks in the BS may also be ascribed to the difference in the amino acid composition or fine structure, ${ }^{32}$ or to hardening because of water evaporation from spider draglines. As prolonged exposure to $\mathrm{UV}-\mathrm{A}^{\star}$ and $\mathrm{UV}-\mathrm{B}^{\star}$ irradiation significantly changed the BS, spiders could not trust the mechanical strength above the ES.

The probability of the breaking of draglines becomes very high in the nonlinear region. In particular, the BS changed markedly under UV irradiation. Thus, spiders could not trust all the mechanical functions of the nonlinear region and also the BS as they cannot know when and how the mechanical function changes rapidly depending on environmental factors such as weather.

In contrast, ES and the slope of the straight line in the stress-strain curve remained almost constant even under $U V-A^{*}$ and $U V-B^{*}$ irradiation throughout the remainder of the extended period investigated (see Figures 3a, b and 4). At the same time, the value of ELS remained almost twice that of the spider's weight, corresponding to the concept of maximum safety. Thus, spiders could trust the ES during the long period of UV irradiation investigation, as they can estimate the limit in the linear region of the mechanical function exactly even under the restrict condition. The results of this experiment strongly supported the argument that spiders could trust mechanically the linear region but not the nonlinear region of the stress-strain curve.

\section{Trust in lifelines deeper than other silks}

Draglines consist of two filaments, each capable of supporting the spider's weight. As the ELS of a dragline corresponds to twice the

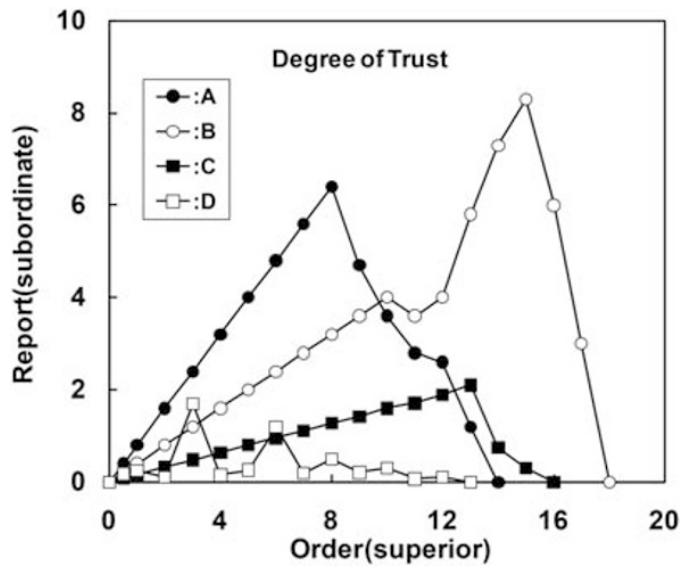

Figure 5 The reports presented by subordinates plotted against the orders given by a superior. Trust can be established between the superior and subordinate when the order-report relationship is linear. A higher slope reflects a higher degree of trust. The rank of evaluation for routine work is in the order of subordinates $\mathrm{A}, \mathrm{B}$ and $\mathrm{C}$. The superior is unable to trust subordinate $D$, as the level of this subordinate's report is random. Closed circles represent subordinate $A$, open circles represent subordinate $B$, closed squares represent subordinate $C$ and open squares represent subordinate $D$.

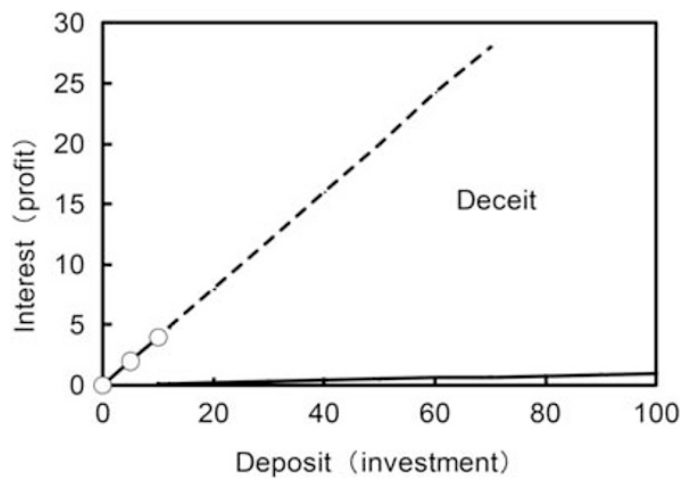

Figure 6 The relationship between interest as a profit and deposit as an investment. The interest is generally very low, even when a large amount of money is deposited (solid line). If there are two or three points (open circles), including an origin, a straight line can be drawn. If an investor makes a large profit through high interest on at least two occasions, they may trust in the linear relationship and estimate that continued investment will produce more profits (broken line). However, the investor may be deceived.

spider's weight as described above, the spider can safely climb its dragline when carrying a captured insect the weight of which is less than its own. ${ }^{19,20}$ However, a spider does not use its dragline for lifting an insect, but winds swathing bands similar to the bundles of draglines around an insect when lifting the insect heavier than its own weight. This means that the spider absolutely does not forget using draglines to keep its life, as the weight of a spider biting an insect heavier than its own weight reaches the nonlinear curve of draglines. This provides evidence that spiders trust neither the mechanical function of swathing bands nor the nonlinear region of the stressstrain curve of draglines.

The results of the mechanical properties of draglines will be applicable to the radial threads constituting orb webs, as draglines and radial threads are secreted from the same ampullate gland. ${ }^{23}$ It is 
essential that the webs of orb-weaving spiders can trap insect prey; this ability is closely related to the mechanical functions of the spider silk constituting the orb webs. If the mechanical function of radial threads constituting orb webs in a similar way as in Figures 3 and 4 is decreased markedly by UV rays, diurnal $N$. clavata and L. blanda spiders cannot capture insect prey in the daytime. Actually $N$. clavata and $L$. blanda spiders have the special mechanism for mechanically strengthening orb webs by using UV rays so that the mechanical function of the orb webs should not decrease for capturing insect prey.

The decrease in mechanical function of the spider's lifeline by UV rays gives the most urgent and important effects for risk directly related to its death, whereas that of the orb webs by UV rays gives effects for the ability to capture insect prey. A spider has to continue using her lifeline during her action on an orb web as she can recognize the importance of her life and differentiate it from other things. The results suggest that spiders have special ability for evaluating the degree of trust in the mechanical functions of silks and can trust only in the linear region of draglines.

\section{CONCLUSION}

Evaluation of trust is very important in fields such as industrial materials and in economic and psychological issues in social sciences. As described above, however, dealing with trust quantitatively is a very difficult problem. Here, the concept of trust was assessed using a spider's lifeline.

This concept of trust from a spider's lifeline may be applicable to specifications for various industrial materials such as fibers, textiles and plastics and also to our future lives. Usually we have to evaluate the quality of industrial materials by outdoor exposure to UV rays. The quality of materials is generally determined from the mechanical properties in the linear region of the materials. In fact, we trust the mechanical properties in the linear region of materials.

Thus, the ELS and ES expressing the maximal limit of trust will be added as specifications of mechanical properties for various industrial materials.

On the other hand, this concept is applicable to the field of social science. For example, most details of our lives are generally an extension of our present lives as most of our lives do not change greatly in the short term. Therefore, most elements will progress linearly in the near future, and we can live at ease as we can predict most of the near future. However, our lives often involve a small number of factors with the potential to change markedly and nonlinearly in the near future. Furthermore, it is extremely difficult to accurately predict our lives in the long term because a large part of our lives in the actual world changes markedly and nonlinearly in the distant future. Although we can live in the near future according to conventional thoughts and methods, we will not be able to survive in the long term without new ideas and approaches. Therefore, it is important that we learn and study cultures other than our own to progress even in the nonlinear world of the distant future.

In conclusion, the stress-strain curves of draglines and the effects of UV rays on its mechanical properties gave an origin for evaluating the degree of trust in various environments. The degree of trust was assessed visually by determining whether the relationship between the stimulus and response was linear or nonlinear. The environment could be trusted if a linear relationship could be established, but could not be trusted if the relationship was nonlinear. This concept of trust could be extended to specifications for mechanical properties of industrial materials such as rope, string and films, which are used repeatedly, and also to various social phenomena such as relationships between a superior's orders and a subordinate's reports (see Appendix described below), between an investment and profit, and between a deposit and the interest relating to rules for deceit (see Appendix described below), politics, baseball games and so on. However, evaluating the degree of trust numerically remains an important problem to be resolved.

1 Lord, P. R. Short fibres and quality control. J. Text. Inst. 76, 388-390 (1985).

2 Glaeser, E., Laibson, D. I., Scheinkman, J. \& Soutter, C. Measuring trust. Quarterly J. Econom. 115, 811-846 (2000).

3 Molm, L. D., Takahashi, N. \& Peterson, G. Risk and trust in social exchange: an experimental test of a classical proposition. Am. J. Sociol. 105, 1396-1427 (2000).

4 Alesina, A. \& Eliana, L. F. Who trusts others? J. Publ. Econom. 85, 207-234 (2002).

5 Cook, K. S., Yamagishi, T., Cheshire, C., Cooper, R., Matsuda, M. \& Mashima, R. Trust building taking: a cross-societal experiment. Social Psychol. Quarterly 68, 121-142 (2005).

6 Work, R. W. \& Morosoff, N. S. A physico-chemical study of the supercontraction of spider major ampullate silk fibers. Text. Res. J. 52, 349-356 (1982).

7 Gosline, J. M., Denny, M. W. \& DeMond, M. E. Spider silk as rubber. Nature 309, 551-552 (1984)

8 Osaki, S. Aging of spider silks. Acta. Arachnol. 43, 1-4 (1994).

9 Vollrath, F. Biology of spider silk. Int. J. Biol. Macromol. 24, 81-88 (1999).

10 Osaki, S., Yamamoto, K., Kajiwara, A. \& Murata, M. Evaluation of the resistance of spider silk to ultraviolet irradiation. Pol. J. 36, 623-627 (2004).

11 Osaki, S. Ultraviolet rays mechanically strengthen spider's silks. Pol. J. 36, 657-660 (2004).

12 Brandwood, A. Mechanical properties and factors of safety of spider drag-lines. J. Exp. Biol. 116, 141-151 (1985).

13 Becker, M. A., Mahoney, D. V., Lenhert, P. G., Eby, R. K., Kaplan, D. \& Adams, W. W. in Silk Polymers (eds Kaplan, D. et al.) 185-195 (American Chemical Society Washington, DC, 1994).

14 Cunniff, P. M., Fossey, S. A., Auerbach, M. A. \& Song, J. W. in Silk Polymers (ACS Symposium Series 544) (eds Kaplan, D. et al.), 234-251 (American Chemical Society: Washington, DC, 1994).

15 Termonia, Y. in Structural Biological Materials (ed. Elices, M.), 335 (Pergamon Press, Amsterdam, 2000)

16 Perez-Rigueiro, J., Elices, M., Llorca, J. \& Viney, C. Tensile properties of Argiope trifasciata drag line silk obtained from spider's web. J. Appl. Polym. Sci. 82, 2245-2251 (2001).

17 Osaki, S. \& Ishikawa, R. Determination of elastic modulus of spider's silks. Pol. J. 34 25-29 (2002)

18 Osaki, S. in Macromolecular Nanostructured Materials (eds Ueyama N. \& Harada, A.), 297-320 (Kodansha Springer, Berlin Heidelberg, 2004).

19 Osaki, S. Spider silk as mechanical lifeline. Nature 348, 419 (1996).

20 Osaki, S. Is the mechanical strength of spider's drag-lines reasonable as lifeline? Int. J. Biol. Macro. 24, 283-287 (1999).

21 Osaki, S. Safety coefficient of the mechanical lifeline of spiders. Pol. J. 35, 261-265 (2003).

22 Osaki, S. Allowable mechanical stress applied to a spider's lifeline. Pol. J. 39, 267-270 (2007).

23 Osaki, S. Thermal properties of spider's thread. Acta. Arachnol. 37, 69-75 (1989).

24 Foelix, R. E. Biology of Spiders 109 (Harvard University Press Cambridge, England, 1982).

25 Hayashi, C. Y., Shipley, N. H. \& Lewis, R. V. Hypotheses that correlate the sequence, structure, and mechanical properties of spider silk proteins. Int. J. Biol. Macromol. 24, 271-275 (1999).

26 Scheibel, T. Spider silks: recombinant synthesis, assembly, spinning, and engineering of synthetic proteins. Microb. Cell Fact. 3, 14-24 (2004).

27 Osaki, S. \& Osaki, M. Evolution of spiders from nocturnal to diurnal gave spider silks mechanical resistance against UV irradiation. Polym. J. (e-pub ahead of print 8 December 2010; doi:10.1038/pj:2010.119).

28 Grobbelaar, C. J., du Plessis, T. A. \& Marais, F. The radiation improvement of polyethyelene prostheses. A preliminary study. J. Bone Joint Surg. 60-B, 370-374 (1987).

29 Yokota, R. Mechanical properties: increase in mechanical properties ascribed to high polymerization. Kobunshi (in Japanese). 39, 371 (1990).

30 Nishikubo, T., Kameyama, A., Tsutsui, K. \& Iyo, M. Synthesis and photochemical reaction of novel p-alkylcalix[6]arene. J. Polym. Sci. Part A Polym. Chem. 37, 1805-1814 (1999).

31 Marshall, I. \& Todd, A. The thermal degradation of polyethylene terephthalate. Trans. Faraday Soc. 49, 67-78 (1953).

32 Matsuhira, T., Yamamoto, K. \& Osaki, S. UV-resistivity of spider silk. Polym. Prept. Jpn. 59: NO 11887 (2010). 


\section{APPENDIX}

\section{RELATIONSHIP BETWEEN STIMULUS AND RESPONSE}

In this model system, subordinates present their reports as the response, and the superior gives different types of orders with different grades to the subordinates as the stimulus. The reports presented by subordinates are plotted against the orders given by a superior. The superior is required to estimate the relative difficulty of routine tasks and rank the orders. The rank is usually estimated numerically from the number of routine tasks. Of course, the superior also has to provide a rank by assessing the level of quality of the tasks as accurate as possible even though numerical estimation is very difficult. This assessment is usually carried out in companies. The superior checks whether the relationship between the order and the report is linear, and evaluates the degree of trust in the subordinates from the slope and length of the straight line in the graph.

\section{Relationship between superior and subordinate}

The concept of trust developed through the study of the mechanical behavior of draglines may be applicable to various kinds of social phenomena. Here, a case in which a superior orders several subordinates to perform tasks at different levels of difficulty is considered. Even though the subordinates try to complete the work, they may not be able to achieve the level required by the superior. Generally, the superior cannot trust in the subordinate until the subordinate presents the reports expected. Therefore, a linear relationship will be established, within error, between the order and the report if there are several incidents in which the level of the report approximates the level of the superior's order. Generally, it is relatively easy to estimate numerically the degree of level, not for research, but for routine work. If linear behavior in the order-report relationship is established for routine work, as shown in cases A, B and C of Figure 5, the superior will trust the subordinate. The longer the straight line, the more widely the subordinate will be trusted by the superior, and the greater the slope of the line the higher the degree of trust. A greater slope means that the subordinate always presents effective results from the superior's order.

Nonlinearity will be established between the superior's order and the subordinate's report for routine tasks if the level of the report is smaller than the level of expectation; as a result, the superior will not trust the subordinate. It is likely that nonlinearity will be established for difficult or unfamiliar tasks, which usually include surplus workload and research. Generally, a nonlinear relationship will be established in the region where the stimulus is very large and the position of the coordinate is very far from the origin.

The rank of evaluation for routine work in Figure 5 is in the order of subordinates A, B and C. However, the length of the linear region and the magnitude of the slope will change depending on the identity of the superior.

The case of $\mathrm{D}$, in which the level of the subordinate's report is random with respect to order, corresponds to the example in which the subordinate does not have the ability to accomplish routine work or does not make appropriate efforts.

Sometimes, subordinates may have a special ability for accomplishing difficult work with a high level in the nonlinear region of the graph, as shown in cases A, B and C in Figure 5. However, most people do not have this ability. As a result, the rank of evaluation made by the superior may reverse for difficult or non-routine work such as research. Most people are unable to easily establish a linear relationship between a superior's order and subordinate's report for difficult research.

This notion of trust is also applicable to cases of learning and deceit, from the viewpoint of investment and profit. It can be assumed that investing money in schooling usually leads to profit through achievement. If profit increases linearly with increasing investment, many people will study hard. If the stream of future society is easily estimated in a similar way, people will also work hard. However, if the future is uncertain and cannot be perfectly estimated, linearity will not be established between effort and profit. As a result, people will make no effort to learn or to work hard.

\section{RULES FOR DECEIT}

In the second example, if the annual interest at a bank is usually very low, people will not expect to earn large sums when depositing small amounts of money (see Figure 6). However, one or two experiences of earning high profits from a small deposit, as a result of high interest, may alter their expectations. If there are two or three points, including an origin, a straight line can be drawn between the deposit and the interest. In such a case, people may believe that a linear relationship has been established, even far from the origin, and continue to invest, and, as a result, be easily deceived. 